Conclusions This study has shown that road traffic Injuries are responsible for most of the maxillofacial fractures. Non-helmet usage among two wheeler users being the most common factor, which needs to be addressed through education and enforcement.

\section{CHALLENGES IN INJURY DATA ENTRY MANAGEMENT IN LOW RESOURCE SETTINGS - EXPERIENCES FROM BANGLADESH}

Afrida Unjum, Jahangir Hossain, Saidur Rahman Mashreky, Fazlur Rahman, Aminur Rahman. Centre for Injury Prevention and Research, Bangladesh

\subsection{6/injuryprev-2016-042156.1039}

Background Centre for Injury Prevention and Research, Bangladesh (CIPRB) is a leading research organisation in Bangladesh which works with different health related data since 2005. Since its inception, CIPRB developed over 25 different data bases mostly on injury prevention. CIPRB also managed surveillance databases with sample around 1,000,000. There are lots of challenges during data entry using hard copy data collection forms.

Objective The objective of the study was to describe the challenges those were faced during management of data entry in a low resource setting, Bangladesh.

Methodology Since 2005 Centre for Injury Prevention and Research, Bangladesh (CIPRB) has been implementing several research programs. CIPRB established a data entry centre in Dhaka with around 30 data enterers. Before starting data entry all hard copy questionnaires were collected and stored in a store room then the data enterers entered injury data manually in a software. The data entry process was closely monitored under the supervision of a statistician. On completion of entry each data-sets was cleaned for analysis.

Results Computers used for data entry were old versioned and unupgraded. Unstable power supply interrupted data entry process. Maintaining network and server system is expensive and time consuming. Frequent damage of the computer sometimes caused loss of entered data. Poor storage system also caused data loss. Missing and confusing unclear data delays entry process. Data entry became slower in the evening than in the morning time. Due to unavailability of spear computers sometimes data enterers needed to wait until fixation of the broken computer.

Conclusion A lot of challenges were identified, which were mostly due to financial constraints. These challenges are common in the low- and middle-income countries. During research funding donors should support to improve the data entry process which will eventually help to conduct better research.

Acknowledgement Centre for Injury Prevention and Research, Bangladesh (CIPRB)

\section{INNOVATIONS IN HOSPITAL-BASED PARTNERSHIPS IN SAFETY AND INJURY PREVENTION: DEVELOPING INTERDEPARTMENTAL COLLABORATIONS}

Helen Arbogast, Santiago Chambers. Children's Hospital Los Angeles

10.1136/injuryprev-2016-042156.1040

Background Injury prevention professionals are part of a complicated system that works collectively to address injury prevention through development, implementation, participation in and evaluation of an injury prevention program. While the responsibilities of an Injury Prevention Manager (IPM) are vast, and often accompanied by minimal support or funding - the need for extensive reach of injury prevention programs remain. Understanding the causes for injury and prevention frameworks, theories, curriculum development, evaluation, research and program implementation are critical for both the departments success in addressing injury prevention and safety campus-wide.

Methods We conducted a gap analysis of safety and injury prevention measures in the hospital to include internal/external outreach and educational needs, programmatic capacity and staffing deficits. We determined the parameters of injury prevention manager's and safety officer's roles in cross fertilisation and involvement in activities based on a review of national norms, focus groups and key informant interviews. We also convened hospitalbased taskforce and community stakeholders to refine the roles and scope of injury prevention priorities for the hospital.

Results Based on these findings, program priorities were set to reflect the following:

- Develop innovative approaches utilising non-traditional partnerships and networks;

- Engage Office of Safety and Security to participate in related trainings and obtain required certifications in injury prevention;

- Collaborate with the Office of Safety and Security on injury prevention internal activities to include patient safety measures (child passenger safety), staff safety, (seat belt and pedestrian safety) and environmental modifications/ enhancements to improve safety/injury prevention capacity (resources, trainings)

- Develop new systems to provide childhood safety information to patients and families;

- Develop creative strategies and partnerships to address resource gaps that will provide program support externally

Conclusions While the Injury Prevention Manager is responsible for developing, implementing and identifying injury prevention programming for patients, families and community; we believe that enhancing injury prevention occurs through sharing best practices and programs and building meaningful partnerships to sustain programmatic reach, establish policy and practice and improve patient and community education. Unfortunately, the impact/reach of these internal partnerships are often limited to hospital-based initiatives if external/community-based priorities unsupported. Elevating the magnitude and critical nature of injury prevention through interdepartmental engagement to secure ongoing support are among the highest priorities for injury prevention managers, particularly trauma centres.

The unique partnership with the Injury Prevention Department and Office of Safety and Security yielded significant outcomes for both the hospital and community-at-large. As a result, more than 8 members from both departments have successfully completed reciprocal trainings to work in concert as Child Passenger Safety Technicians and in disaster training and response to improve the health and safety of patients and families in the community. This interdepartmental collaboration has yielded more than 25 community-based events, 3 internal hospital policies, 6 certification courses and more than 60,000 contacts. Both areas have fully immersed activities and policies to support this partnership long-term. 